\title{
Cortinarius hesleri (Basidiomycota, Agaricales, Cortinariaceae), primer registro para México
}

\section{Cortinarius hesleri (Basidiomycota, Agaricales, Cortinariaceae), first record for Mexico}

\section{Acta Botanica Mexicana}

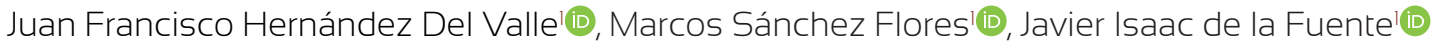 \\ Eduardo Manolo Medrano Zapata'[D, Jesús García Jiménez',2]
}

\section{Resumen:}

Antecedentes y Objetivos: El género Cortinarius está constituido por hongos ectomicorrícicos caracterizados por un velo parcial a modo de "cortina" aracnoide, esporada, color marrón óxido a marrón anaranjado ocráceo y basidiosporas rugosas, arrugadas o verrugosas y sin poro apical. Cortinarius hesleri se ubica en el subgénero Telamonia; se reconoce principalmente por sus basidiomas de colores que abarcan desde los tonos rojos a los anaranjados. Se encuentra distribuida en Norteamérica en Canadá y los Estados Unidos de América. Los inventarios fúngicos facilitan el reconocimiento de especies para una determinada área geográfica. El objetivo de este trabajo es presentar a C. hesleri como primer registro para México.

Métodos: Se recolectaron especímenes en los bosques de Quercus del municipio Victoria en el estado de Tamaulipas, México. Se elaboró una descripción macro y micromorfólogica de la especie, así como láminas con fotografías macroscópicas in situ y de las estructuras microscópicas, que a su vez se ilustraron, para su posterior análisis e identificación taxonómica mediante la literatura especializada correspondiente. Los ejemplares estudiados se depositaron en el herbario micológico "José Castillo Tovar" del Instituto Tecnológico de Ciudad Victoria.

Resultados clave: Los especímenes estudiados cumplen con las características de la descripción original de la especie, así mismo con las del hábitat al que está asociado y en el cual se distribuye. Cortinarius hesleri es descrita por primera vez para México. Se caracteriza por sus basidiomas de color anaranjado, esporas ornamentadas y elipsoides de (7-)8-10(-12) × (5-)5.5-7 $\mu \mathrm{m}$ y por su asociación con especies vegetales del bosque mixto de Quercus-Pinus.

Conclusiones: Cortinarius hesleri se encuentra distribuido ampliamente desde la parte norte y este de Estados Unidos de América, alcanzando su distribución más meridional actualmente conocida en el estado de Tamaulipas en México. Por su potencial asociación ectomicorrícica con especies forestales de los géneros Quercus, Pinus y Carya, C. hesleri pudiera encontrarse distribuido junto a estas especies de plantas en otros sitios del noreste y la parte oriental hacia el sur de México.

Palabras clave: Agaricales, ectomicorriza, fungi, macrohongos, Tamaulipas, Telamonia.

\section{Abstract:}

Background and Aims: The genus Cortinarius consists of ectomycorrhizal fungi, characterized by a partial veil, forming an arachnoid "curtain", spore print rust brown to orange-orange brown, and basidiospores rough, wrinkled or warty and without apical pore. Cortinarius hesleri is placed in the subgenus Telamonia, and can be recognized mainly by its basidiomas with colors from red to orange tones. Fungal inventories facilitate the recognition of species for a specific geographic area. The aim of this work is to present $C$. hesleri as a first record for Mexico.

Methods: The specimens were collected in Quercus forests from the municipality of Victoria, in the state of Tamaulipas. A macro- and micromorphological description of the species was prepared, as well as plates with macroscopic photographs in situ and microscopic structures, which were illustrated, for subsequent analysis and taxonomic identification with the corresponding specialized literature. The studied specimens were deposited in the mycological herbarium "José Castillo Tovar" from the Instituto Tecnológico de Ciudad Victoria.

Key results: The studied specimens meet the characteristics of the original description of the species, as well as those of the habitat to which it is associated and in which it is distributed. Cortinarius hesleri is described for the first time for Mexico. This species is characterized by its orange basidiomata, ornate ellipsoid spores of (7-)8-10(-12) × (5-)5.5-7 $\mu \mathrm{m}$ and its association with plant species from the Quercus-Pinus mixed forest.

Conclusions: Cortinarius hesleri is widely distributed from the northern and eastern part of the United States of America, reaching its southernmost distribution known so far in the state of Tamaulipas, in Mexico. Due to its potential ectomycorrhizal association with forest species of the genera Quercus, Pinus and Carya, C. hesleri could be found distributed with these plant species in other sites in the northeast and the eastern part of Mexico. Key words: Agaricales, ectomycorrhiza, fungi, macrofungi, Tamaulipas, Telamonia.

${ }^{1}$ Instituto Tecnológico de Ciudad Victoria, Tecnológico Nacional de México, Herbario Micológico José CastiIlo Tovar, Boulevard Emilio Portes Gil No. 1301, 87010 Ciudad Victoria, Tamaulipas, México.

${ }^{2}$ Autor para la correspondencia: jgarjim@yahoo. com.mx
Recibido: 2 de diciembre de 2021.

Revisado: 17 de enero de 2022

Aceptado por Marie-Stéphanie Samain: 16 de febrero de 2022.

Publicado Primero en línea: 23 de febrero de 2022.

Publicado: Acta Botanica Mexicana 129(2022).
Citar como: Hernández Del Valle, J. J., M. Sánchez Flores, J. I. de la Fuente, E. M. Medrano Zapata y J. García Jiménez. 2022. Cortinarius hesleri (Basidiomycota, Agaricales, Cortinariaceae), primer registro para México Acta Botanica Mexicana 129: e1927. DOI: https://doi.org/10.21829/abm129.2022.1927 (i) (5) Este es un artículo de acceso abierto bajo la licencia Creative Commons 4.0 Atribución-No Comercial (CC BY-NC 4.0 Internacional).

\section{e-ISSN: 2448-7589}




\section{Introducción}

El género Cortinarius (Pers.) Gray es un grupo de hongos terrestres, cosmopolitas, de hábito ectomicorrícico, caracterizado por un velo parcial típicamente a modo de "cortina" aracnoide, dejando una zona anular, delgada y fibrosa. La esporada es de color marrón óxido a marrón anaranjado ocráceo, y las basidiosporas son rugosas, arrugadas o verrugosas y sin poro apical (Bessette et al., 1997; Rinaldi et al., 2008; Tedersoo et al., 2010). El género pertenece a la familia Cortinariaceae dentro del Orden Agaricales de la clase Agaricomycetes del Phylum Basidiomycota (He et al., 2019) y está constituido por aproximadamente 2250 especies en todo el mundo (He et al., 2019; Wijayawardene et al., 2020; Index Fungorum, 2021); de estas, 500 especies ocurren en América del Norte (Bessette et al., 1997). En México se han registrado, hasta el momento, solo 26 especies del género Cortinarius en los siguientes estados: Aguascalientes, Baja California, Chiapas, Chihuahua, Coahuila, Durango, Jalisco, México, Michoacán, Nuevo León, Tamaulipas, Sonora y Veracruz (Díaz-Barriga et al., 1988; Pardave Díaz, 1996; Garza et al., 2002; García-Jiménez y Guevara-Guerrero, 2005; García y Valenzuela, 2005; Pérez-Silva et al., 2008; Frutis Molina y Valenzuela, 2009; Guzmán et al., 2009; Pérez-Silva et al., 2011; Burrola-Aguilar et al., 2012; García-Jiménez et al., 2014; Domínguez Romero et al., 2015; Padilla-Velarde et al., 2016; Baeza-Guzmán et al., 2017; Kong, et al., 2018; Rodríguez-Alcántar et al., 2018; Garza-Ocañas et al., 2019).

Cortinarius hesleri Ammirati, Niskanen, Liimat. \& Matheny se ubica en el subgénero Telamonia (Fr.) Trog; se caracteriza principalmente porque sus basidiomas presentan una gama de colores que abarca tonos del rojo al anaranjado, como son el rojo marrón (rojo de Brasil), rojo cinabrio, rojo marrón (siena tostado), naranja marrón (naranja marte) o naranja rojizo a naranja marrón (naranja rufo o herrumbre a naranja de xantina) o más naranja a naranja profundo, y generalmente de color más pálido en el margen (Ammirati et al., 2013). La especie se distribuye en bosques mixtos de latifoliadas y coníferas en el este de América del Norte (Ammirati et al., 2013). Como parte de los estudios de la funga del norte de México, en los bosques de Quercus en el estado de Tamaulipas se recolectaron varios especímenes con las características de
Cortinarius hesleri. El objetivo del presente estudio es describir por primera vez a Cortinarius hesleri para México, incluyendo una descripción macro y micromorfólogica de la especie, así como láminas con fotografías macroscópicas in situ, e ilustraciones y fotografías de sus estructuras microscópicas.

\section{Materiales y Métodos}

Se realizaron exploraciones micológicas en la temporada de lluvias durante los meses de agosto a octubre de 2019 y 2020. El sitio de muestreo se ubicó en el bosque de la localidad Sierra Madre, en el municipio Victoria en el estado de Tamaulipas, con coordenadas $23^{\circ} 37^{\prime} 20.10^{\prime \prime} \mathrm{N}, 99^{\circ} 14^{\prime} 37.90^{\prime \prime} \mathrm{O}$ y una altitud de 1650 m (Fig. 1). La vegetación dominante en los sitios de muestreo corresponde a bosque de Quercus y las especies que lo conforman son Quercus canbyi Trel., Q. clivicola Trel. \& C.H. Müll., Q. graciliramis C.H. Mull., Q. obtusata Bonpl. Q. porphyrogenita Trel., Q. rysophylla Weath., Q. sartorii Liebm., Q. sebifera Trel. y Q. xalapensis Bonpl., además de algunos elementos entremezclados de Pinus teocote Schiede ex Schltdl. \& Cham. y Carya ovata (Mill.) K. Koch var. mexicana (Engelm. ex Hemsl.) W.E. Manning (García-Morales et al., 2014 a,b; García-Morales, 2016).

Se recolectaron y registraron ejemplares fúngicos frescos y se preservaron mediante su deshidratación siguiendo los métodos de muestreo para macromicetos propuestos por Lodge et al. (2004). El color de los ejemplares en fresco fue descrito según Kornerup y Wanscher (1978). Para el análisis microscópico de los especímenes se realizaron cortes histológicos hechos a mano de algunas secciones del basidioma deshidratado, con el fin de elaborar preparaciones usando $\mathrm{KOH}$ al 5\% (hidróxido de potasio), $\mathrm{NH}_{4} \mathrm{OH}$ (hidróxido de amonio) y reactivo de $\mathrm{Me}$ Izer. Las preparaciones se observaron en un microscopio óptico (Axiostar plus, Carl Zeiss, Jena, Alemania) (Largent et al., 1977). El tamaño de los microcaracteres, tales como basidios, cistidios y basidiosporas, se determinó midiendo 30 elementos de cada uno. Las abreviaturas utilizadas son: $\mathrm{Q}=$ relación longitud/ancho de las basidiosporas; $\mathrm{L}=$ longitud promedio; $\mathrm{W}=$ =ancho promedio; $\mathrm{N}=$ total de esporas medidas. 


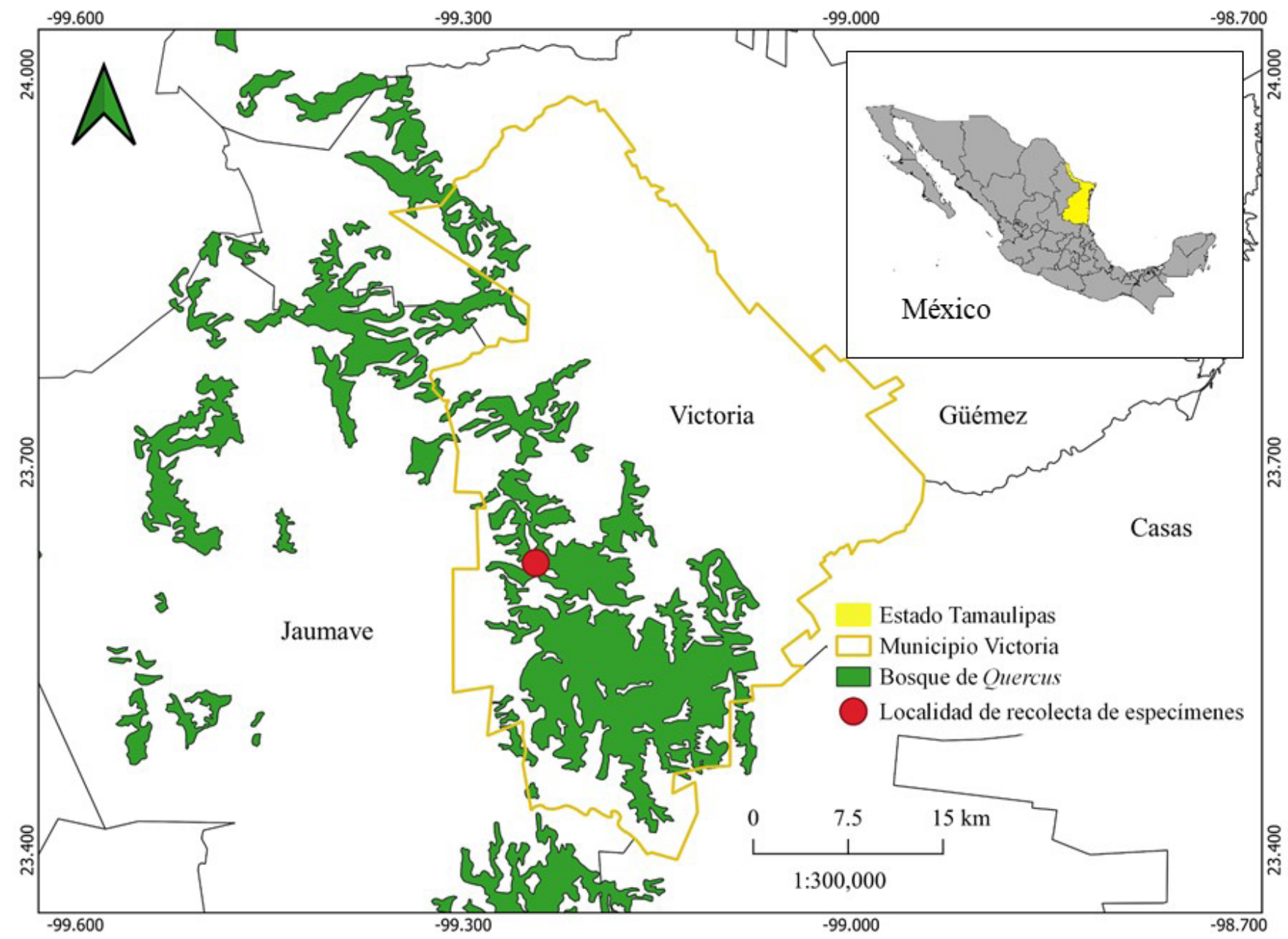

Figura 1: Localidad de recolecta de especímenes de Cortinarius hesleri Ammirati, Niskanen, Liimat. \& Mathenyen, en Tamaulipas, México.

Para la identificación de la especie se revisó literatura especializada (Ammirati, 1972; Ammirati et al., 2013), para indicar el estatus taxonómico de la especie estudiada se revisó el Index Fungorum (2021), mientras que para la autoría de las especies vegetales se revisó Plants of the World (2021). Todos los especímenes fueron herborizados y depositados en el herbario micológico "José Castillo Tovar" del Instituto Tecnológico de Ciudad Victoria (ITCV).

El mapa para la ubicación del sitio de muestreo se elaboró en el programa cumputacional QGIS v. 3.22.3 (QGIS Development Team, 2022). Las fotografías de los especímenes en fresco se realizaron con una cámara Sony (DSCHX60V, Sony Corporation, Tokio, Japón). Las estructuras microscópicas se ilustraron a partir de dibujos realizados a mano alzada para su posterior digitalización.

\section{Resultados}

Cortinarius hesleri Ammirati, Niskanen, Liimat. \& Matheny, Botany 91: 96. 2013. Figs. 2, 3, 4.

TIPO: ESTADOS UNIDOS DE AMÉRICA. Michigan, Washtenaw, Gorman Lake, 19.VIII.1972, A. H. Smith 81565 (holotipo: $\mathrm{MICH}$ !).

Píleo 11-50 mm de diámetro, cónico, cónico campanulado, umbonado a convexo umbonado, superficie lisa, algo sedosa brillante, color anaranjado pálido (5A5) a anaranjado profundo (5A8) cuando joven, anaranjado marrón (5C5) al centro, anaranjado pálido al margen (5A3) en la madurez; margen enrollado cuando joven, lue- 

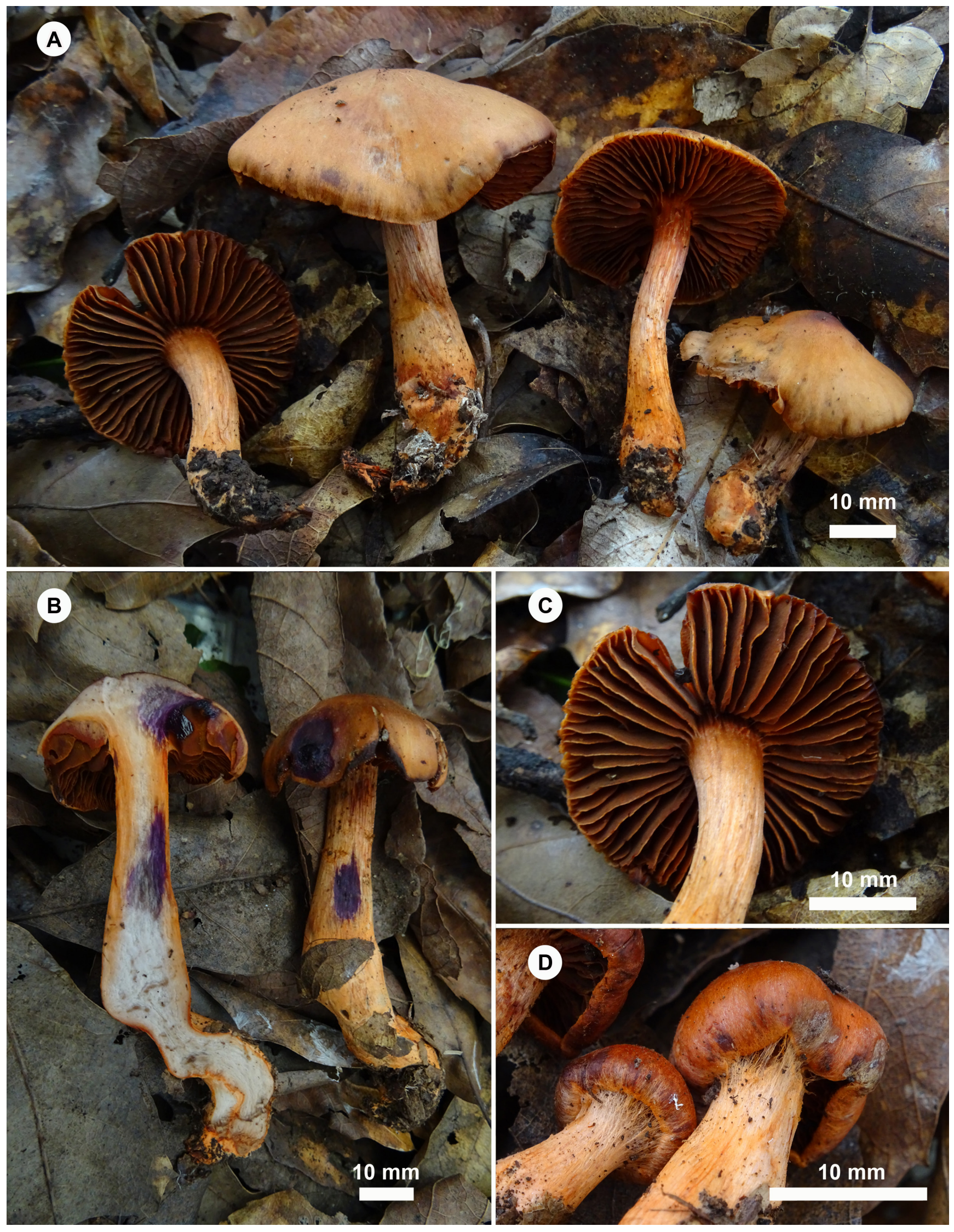

Figura 2: Cortinarius hesleri Ammirati, Niskanen, Liimat. \& Matheny. A. basidiomas in situ; B. corte longitudinal de basidiomas y reacciones químicas color púrpura-violáceo con $\mathrm{KOH}$ al 5\%; C. acercamiento a láminas; D. cortina a modo de restos membranosos del margen del píleo al estípite en basidiomas en desarrollo. 
A

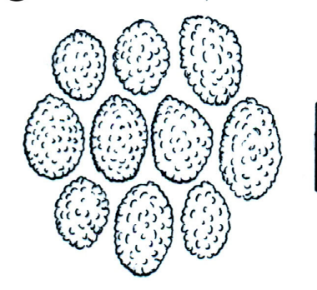

B

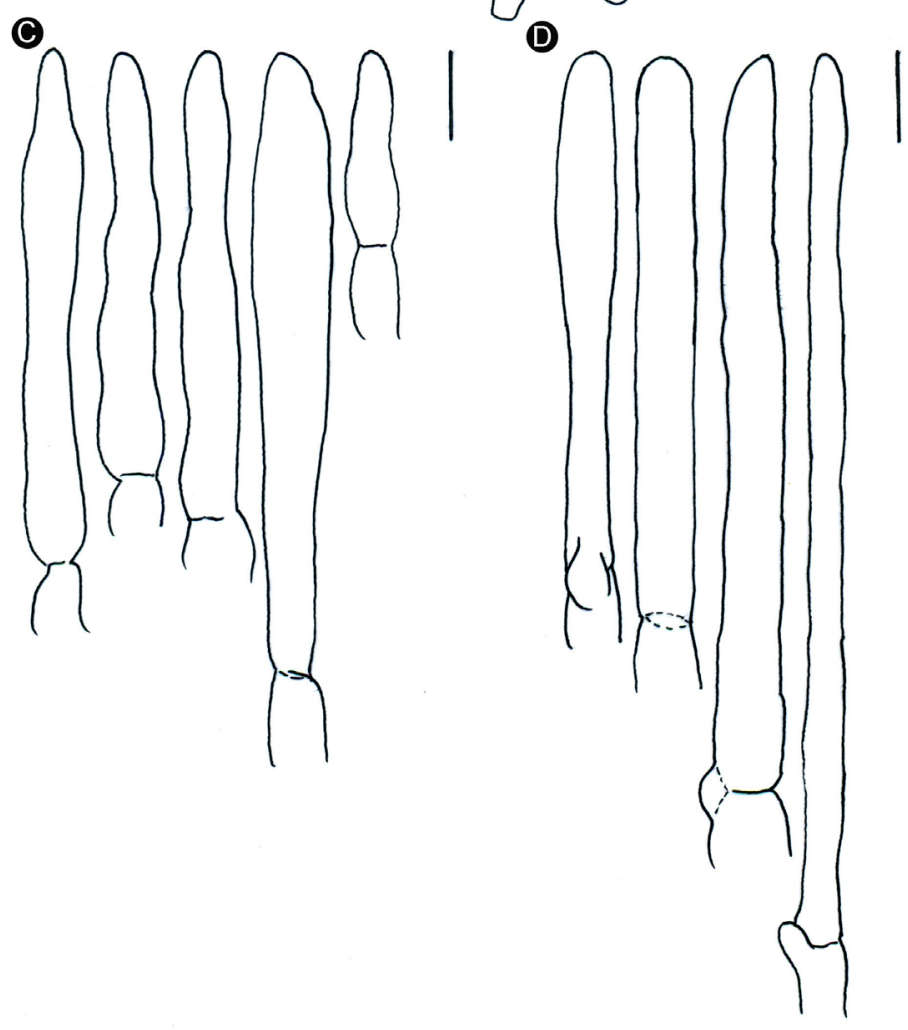

Figura 3: Cortinarius hesleri Ammirati, Niskanen, Liimat. \& Matheny. Ilustración de estructuras microscópicas. A. basidiosporas; B. basidios; C. pileipellis, hifas terminales; D. estipitipellis, hifas terminales. Escala=10 $\mu \mathrm{m}$.

go entero-ondulado cuando maduro y agrietándose cuando viejo, al aplicar $\mathrm{NH}_{4} \mathrm{OH}$ y $\mathrm{KOH}$ al $5 \%$ se torna de violeta vívido (17A8) a violeta oscuro (17A7); láminas sinuadas a subadheridas, 5-10 $\mathrm{mm}$ de ancho, color rojo amarillento (8A8) anaranjado grisáceo a anaranjado (6B5-7) al borde, más o menos distantes (hasta $1 \mathrm{~mm}$ ) y gruesas, borde ondulado, algo erosionado irregular, con lamélulas, se tornan violeta grisáceo (17D-5) con $\mathrm{KOH}$ al $5 \%$ y $\mathrm{NH}_{4} \mathrm{OH}$; estípite 23-90 × 5-12 mm, subcilíndrico ensanchándose a la base, algo curvo, superficie longitudinalmente fibrilosa, color anaranjado brillante (5A5), con algunos finos restos membranosos a modo de velo o fibrillas longitudinales color anaranjado (5A7-6) o anaranjado profundo (6A8), más notable en ejemplares jóvenes, seco, sedoso, brillante, consistencia fibrosa, compacto; base con micelio anaranjado (5A6) el cual perdura en ejemplares herborizados, al aplicar $\mathrm{NH}_{4} \mathrm{OH}$ y $\mathrm{KOH}$ al $5 \%$ se torna violeta vívido (17A8) a violeta oscuro (17A7); contexto 2-9 mm de grosor; compacto; color blanquecino grisáceo con tonos anaranjados (5A2) en el estípite, anaranjado rojizo brillante (5A7) hacia la superficie (cutícula), se torna violeta vívido (17A8) al reaccionar con $\mathrm{NH}_{4} \mathrm{OH}$ y $\mathrm{KOH}$ al $5 \%$; olor fúngico indistinguible; basidiosporas (7-)8-10(-12) $\times(5-) 5.5$ $7 \mu \mathrm{m} ; \mathrm{Q}=1.46-1.49 ; L^{\prime}=8.72, \mathrm{~W}^{\prime}=6.05, \mathrm{~N}=30$, elipsoides, raramente ampliamente elipsoide o elongada (oblonga), amigdaloides, verrugosa a ligeramente verrugosas (ornamentación poco visible), color marrón a marrón amarillento en $\mathrm{KOH}$ al 5\%, dextrinoides (marrón oscuro) en Melzer, pared delgada, apículo no visible; basidios 28-39 $\times 7-9.5 \mu \mathrm{m}$, clavados, hialinos en $\mathrm{KOH}$ al 5\%, tetraspóricos, pared gruesa, gutulados, con fíbulas en la base; queilocistidios y pleurocistidios ausentes; subhimenio no diferenciado; trama himenoforal paralela regular, formada por hifas septadas, cilíndricas, 5-12 $\mu \mathrm{m}$ de diámetro, hialinas con algunos tonos marrón-rojizos en $\mathrm{KOH}$ al $5 \%$, pared delgada; pileipellis formado por un cutis, con elementos terminales $22.5-72 \times 6.5-11 \mu \mathrm{m}$, ápice redondeado a obtuso; hifas cilíndricas a ligeramente entrelazadas, hialinas en $\mathrm{KOH}$ al $5 \%$, algunas con pigmentos marrón rojizo (8C7), pared delgada, en algunas se observa la presencia de fíbulas; estipitipellis con elementos terminales postrados 61$102 \times 4.5-8 \mu \mathrm{m}$, ápice redondeado; hifas cilíndricas, hialinas a ligeramente amarillento en $\mathrm{KOH}$ al $5 \%$, algunas con pigmentos marrón rojizo (8C7), pared delgada, en algunas se observa la presencia de fíbulas.

Hábito y hábitat: especie ectomicorrícica que crece de manera solitaria a gregaria en pequeños grupos, en ocasiones cespitoso, entre la hojarasca o en el suelo del bosque de Quercus-Pinus, a 1600 m s.n.m. Fructifica en los meses de agosto a octubre.

Distribución: Norteamérica, en Canadá y los Estados Unidos de América (Ammirati, 1972; Ammirati et al., 2013). Se cita por primera vez para México. 

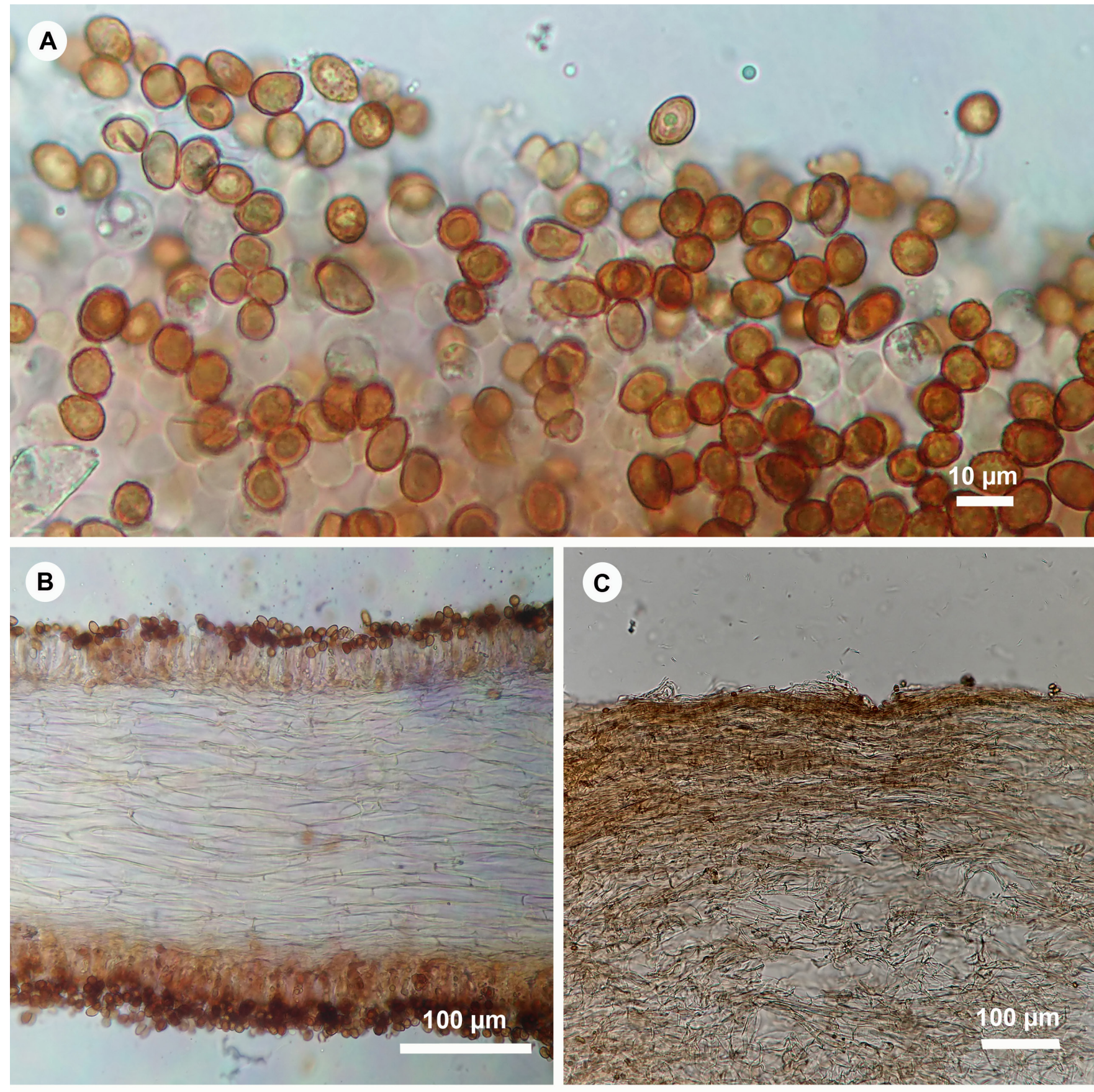

\section{C}
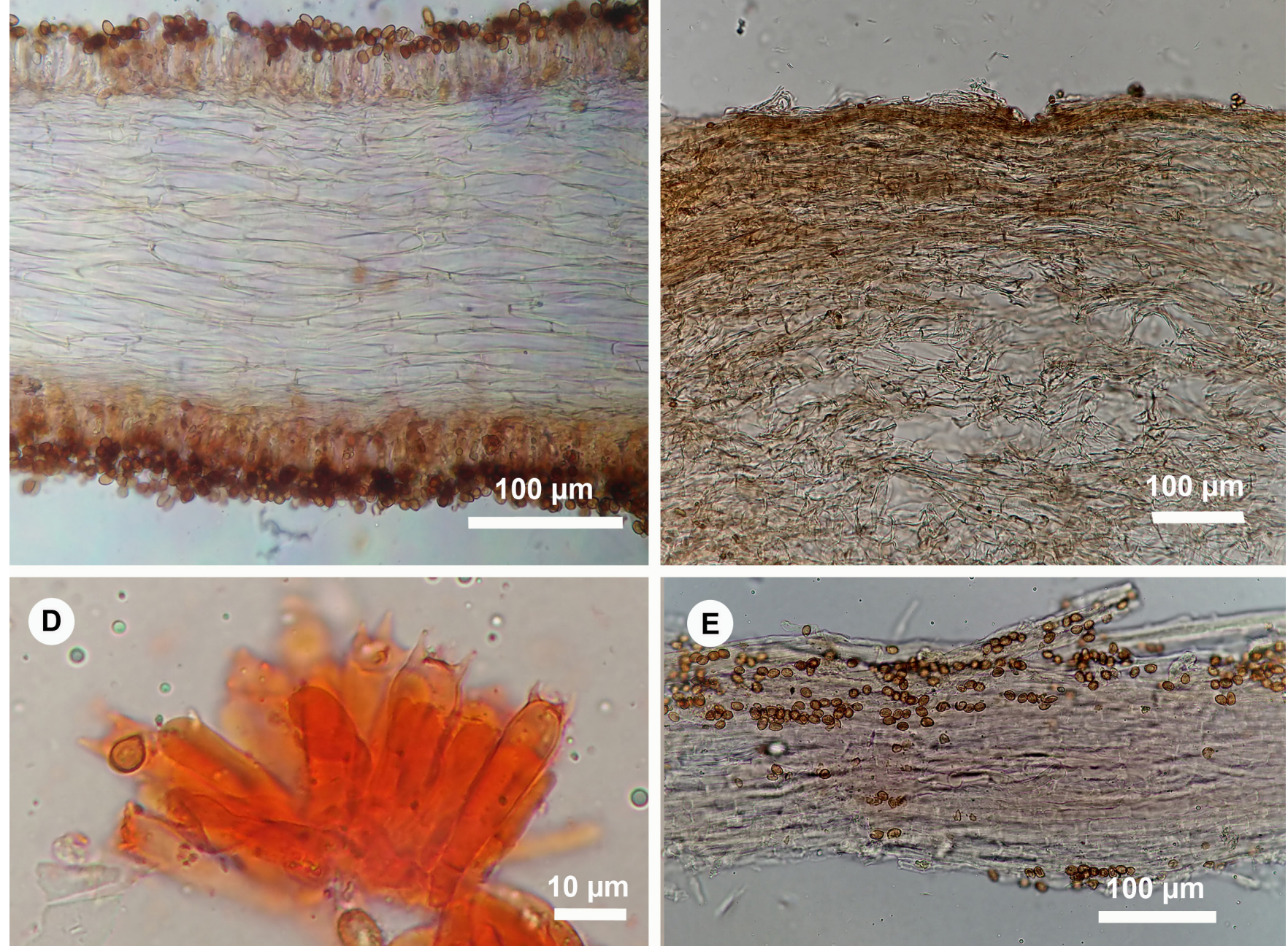

Figura 4: Cortinarius hesleri Ammirati, Niskanen, Liimat. \& Matheny. Estructuras microscópicas. A. basidiosporas; B. trama himenoforal; C. pileipellis; D. basidios; E. estipitipellis. 
Material examinado: MÉXICO. Tamaulipas, municipio Victoria, ejido Sierra Madre, 1650 m, 2337'20.10' N, 99¹4'37.90"O, 24.IX.2019, F. Hernández 208 (ITCV); loc. cit., 10.X.2019, F. Hernández 267 (ITCV); loc. cit., 26.X.2019, F. Hernández 353 (ITCV); loc. cit., 26.VIII.2020, F. Hernández 488 (ITCV).

Notas taxonómicas: las características de los especímenes estudiados coinciden con las proporcionadas de Cortinarius hesleri en la descripción original de Ammirati et al. (2013). Esta especie se encuentra relacionada en América del Norte y dentro del subgénero Telamonia con Cortinarius californicus A.H. Sm., el cual se diferencia por tener esporas más angostas y ligeramente más cortas, 7.79.6(-10.2) × 4.8-5.5(-7) $\mu \mathrm{m}, \mathrm{Q}=1.4-1.9$, siendo elipsoides a elongadas $u$ oblongas, mientras que $C$. hesleri presenta esporas más anchas y largas (7-)8-10(-12) × (5-)5.5-7 $\mu \mathrm{m}$, $\mathrm{Q}=1.46-1.49$, y forma amigdaloide a elipsoide. La reacción química violeta con $\mathrm{KOH}$ al 5\%, que ocurre en el material fresco en cualquiera de sus tejidos, también se presenta en el material seco, observándose incluso a nivel microscópico donde se desprende un pigmento violeta (17A8) que luego se torna marrón rojizo (8C7).

\section{Discusión}

Dentro del subgénero Telamonia se encuentra Cortinarius hesleri junto a C. californicus, C. cinnabarinus Fr. y $C$. coccineus Reumaux, siendo todas estas especies muy afines principalmente por su aspecto de color naranja a rojo anaranjado. Cortinarius hesleri se había relacionado originalmente con $C$. cinnabarinus, por sus similitudes macromorfológicas y la asociación con plantas del género Quercus L. y otras latifoliadas (Keller y Ammirati, 1983). Sin embargo, posteriormente estudios moleculares demostraron que ambas especies son diferentes y que $C$. hesleri está relacionada filogenéticamente de modo más estrecho con C. californicus que con C. cinnabarinus. Además, $C$. cinnabarinus y $C$. coccineus se distribuyen principalmente en bosques europeos (Ammirati et al., 2013).

Cortinarius californicus está reportada en asociación, principalmente, con bosques mixtos de Pinus L., Pseudotsuga Carrière, Thuja L., Arbutus L., Acer L. y Quercus garryana Douglas ex Hook., mientras que $C$. hesleri se re- porta asociada a los bosques mixtos de Quercus-Pinus, Quercus y Carya Nutt. en Canadá y los Estados Unidos de América (Ammirati et al., 2013), lo cual coincide con el tipo de hábitat en el que se encuentra en México. Este se trata del bosque de Quercus mezclado con Pinus teocote y Carya ovata var. mexicana, donde también se presentan Quercus clivicola, Quercus graciliramis, Quercus sartorii y Pinus teocote, con quienes probablemente forma asociaciones ectomicorrícicas.

Cortinarius californicus se encuentra distribuido al oeste de los Estados Unidos de América y al oeste de la Columbia Británica en Vancouver, Canadá, mientras que $C$. hesleri ocurre en la mayor parte del este de América del Norte, desde el norte en Nueva York y Michigan, pasando por Illinois, Virginia, Carolina del Norte, Tennessee, Florida, hasta el sur en Texas (Ammirati, 1972; Keller y Ammirati, 1983; Ammirati et al., 2013). La distribución de C. hesleri ocurre por la zona este hacia el sur de Texas en los Estados Unidos de América y continúa en el noreste de México en el estado de Tamaulipas, siendo esta su distribución más meridional conocida por el momento en el continente americano. Esta distribución coincide con lo comentado por Ammirati et al. (2013), quienes sugieren que $C$. hesleri se encontraría probablemente distribuido de forma extensa en la parte este y sur de América del Norte.

\section{Conclusiones}

Hasta el momento $C$. hesleri cuenta con una distribución muy marcada hacia el oriente de Norteamérica y una asociación muy particular con bosques templados, donde se destacan principalmente por lo menos tres géneros de plantas ectomicorrícicas: Quercus, Pinus y Carya (Smith y Read, 2008; Ammirati et al., 2013). De acuerdo con lo anterior, y reconociendo que el género Cortinarius comprende especies de hábito ectomicorrícico (Rinaldi et al., 2008), se sugiere que $C$. hesleri es una especie que se puede encontrar distribuida de manera extensa en otros sitios del noreste y la parte oriental hacia el sur de México, junto a los bosques y las especies forestales con las que se le ha ubicado anteriormente y con los cuales pudiera tener una asociación ectomicorrícica. Futuros muestreos podrían dilucidar más objetivamente la distribución de la especie. Estudios morfológicos y moleculares sobre la micorriza permitirían 
confirmar la relación simbiótica de $C$. hesleri con sus árboles hospederos.

\section{Contribución de autores}

JFHV, MSF y JGJ estructuraron y diseñaron el estudio. JFHV y JGJ realizaron las recolecciones y determinaciones de los ejemplares. JFHV, MSF y JGJ contribuyeron a la adquisición de información especializada para dicho trabajo. JFHV redactó el manuscrito, tomó las fotografías y elaboró los dibujos, EMMZ realizó el mapa. JFHV, JGJ, MSF y JIF revisaron y aprobaron el manuscrito final.

\section{Financiamiento}

La presente investigación se llevó a cabo con apoyo del Consejo Nacional de Ciencia y Tecnología (CONACYT).

\section{Agradecimientos}

Los autores agradecen al Instituto Tecnológico de Ciudad Victoria.

\section{Literatura citada}

Ammirati, J. F. 1972. The section Dermocybe of Cortinarius in North America. Ph.D. dissertation. University of Michigan. Ann Arbor, USA. 282 pp.

Ammirati, J. F., K. W. Hughes, K. Liimatainen, T. Niskanen y P. B. Matheny. 2013. Cortinarius hesleri from Eastern North America and related species from Europe and western North America. Botany 91(2): 91-98. DOI: https://doi. org/10.1139/cjb-2012-0154

Baeza-Guzmán, Y., R. Medel-Ortiz y R. Garibay-Orijel. 2017. Caracterización morfológica y genética de los hongos ectomicorrízicos asociados a bosques de Pinus hartwegii en el Parque Nacional Cofre de Perote, Veracruz. Revista Mexicana de Biodiversidad 88(1): 41-48. DOI: http://doi. org/10.1016/j.rmb.2017.01.027

Bessette, A. E., A. R. Bessette y D. W. Fischer. 1997. Mushrooms of Northeastern North America. Syracuse University Press. Syracuse, USA. 582 pp.

Burrola-Aguilar, C., O. Montiel, R. Garibay-Orijel y L. Zizumbo-Villarreal. 2012. Conocimiento tradicional y aprovechamiento de los hongos comestibles silvestres en la región de Amanalco, Estado de México. Revista Mexicana de Micología 35: 1-16.
Díaz-Barriga, H., F. Guevara-Fefer y R. Valenzuela. 1988. Contribución al conocimiento de los macromicetos del estado de Michoacán. Acta Botanica Mexicana 2: 21-44. DOI: https://doi.org/10.21829/abm2.1988.564

Domínguez Romero, D., J. I. Arzaluz Reyes, C. Valdés Valdés y N. P. Romero Popoca. 2015. Uso y manejo de hongos silvestres en cinco comunidades del municipio de Ocoyoacac, Estado de México. Tropical and Subtropical Agroecosystems 18(2): 133-143.

Frutis Molina, I. y R. Valenzuela. 2009. Macromicetos. In: Ceballos, G., R. List, G. Garduño, R. López Cano, M. J. Muñozcano Quintanar, E. Collado y J. E. San Román (comps.). La diversidad biológica del Estado de México, Estudio de Estado. Gobierno del Estado de México, Biblioteca Mexiquense del Bicentenario. Toluca, México. Pp. 243-250.

García, J. y R. Valenzuela. 2005. Hongos Macromicetos. In: Sánchez-Ramos, G., P. Reyes-Castillo y R. Dirzo (eds.). Historia Natural de la Reserva de la Biosfera El Cielo, Tamaulipas, México. Universidad Autónoma de Tamaulipas. Victoria, México. Pp. 321-337.

García-Jiménez, J., R. Valenzuela, T. Raymundo, L. J. GarcíaMorales, G. Guevara, F. Garza, E. Cázares y E. R. Cancino. 2014. Macrohongos asociados a encinares (Quercus spp.) en algunas localidades del estado de Tamaulipas, México. In: Correa Sandoval, A., J. V. Horta-Vega, J. García-Jiménez y L. Barrientos Lozano (eds.). Biodiversidad Tamaulipeca, Vol. 2. Instituto Tecnológico de Ciudad Victoria. Victoria, México. 173 pp.

García-Jiménez, J. y G. Guevara-Guerrero. 2005. Macromicetos (hongos superiores) de Tamaulipas. In: Barrientos-Lozano, L., A. Correa Sandoval, J. V. Horta-Vega y J. García-Jiménez (eds.). Biodiversidad Tamaulipeca, Vol. 1. Dirección General de Educación Superior Tecnológica-Instituto Tecnológico de Ciudad Victoria. Victoria, México. Pp. 67-79.

García-Morales, L. J. 2016. Taxonomía y fitogeografía de la familia Fagaceae (Magnoliophyta: Fagales) en los estados de Tamaulipas y Nuevo León, México. Tesis de doctorado. Facultad de Ciencias Forestales, Universidad Autónoma de Nuevo León. Linares, México. 306 pp.

García-Morales, L. J., A. E. Estrada Castillón, J. García Jiménez, J. A. Villarreal Quintanilla, C. Cantú Ayala, E. Jurado Ybarra y V. A. Vargas Vázquez. 2014a. Florística y vegetación del Área 
Natural Protegida Altas Cumbres, Tamaulipas, México. In: Correa Sandoval, A., J. V. Horta-Vega, J. García-Jiménez y L. Barrientos Lozano (eds.). Biodiversidad Tamaulipeca, Vol. 2. Instituto Tecnológico de Ciudad Victoria. Victoria, México. Pp. 15-73.

García-Morales, L. J., A. E. Estrada Castillón, C. Cantú Ayala, E. Jurado Ybarra y J. A. Villarreal Quintanilla. 2014b. Diversidad y conservación de los encinos (Fagaceae) en el Estado de Tamaulipas. In: Correa Sandoval, A., J. V. Horta-Vega, J. García-Jiménez y L. Barrientos Lozano (eds.). Biodiversidad Tamaulipeca, Vol. 2, Núm. 1. Tecnológico Nacional de México-Instituto Tecnológico de Ciudad Victoria. Victoria, México. Pp. 3-13.

Garza, F., J. García, E. Estrada y H. Villalón. 2002. Macromicetos, ectomicorrizas y cultivos de Pinus culminicola en Nuevo León. Ciencia UANL 2(5): 204-210.

Garza-Ocañas, F., M. Quiñónez-Martínez, L. Garza-Ocañas, A. Carrillo-Parra, H. Villalón-Mendoza, H. GonzálezRodríguez, R. Valenzuela-Garza, G. Guevara-Guerrero, J. García-Jiménez y M. García-Aranda. 2019. Some edible, toxic and medicinal mushrooms from temperate forests in the north of Mexico. In: Sridhar, K. R. y S. K. Deshmukh (eds.). Advances in macrofungi. Diversity, Ecology and Biotechnology. Tylor and Francis Group. Boca Raton, USA. Pp. 144-198.

Guzmán, G., R. Medel y F. Ramírez Guillén. 2009. Hongos. In: Ceballos, G., R. List, G. Garduño, R. López Cano, M. J. Muñozcano Quintanar, E. Collado y J. Eivin San Román (comps.). La diversidad biológica del Estado de México. Estudio de Estado. Gobierno del Estado de México, Biblioteca Mexiquense del Bicentenario. Toluca, México. Pp. 251-260.

He M. Q., R. L. Zhao, K. D. Hyde, D. Begerow, M. Kemler, A. Yurkov, E. H. C. McKenzie, O. Raspé, M. Kakishima, S. Sánchez-Ramírez, E. C. Vellinga, R. Halling, V. Papp, I. V. Zmitrovich, B. Buyck, D. Ertz, N. N. Wijayawardene, B. K. Cui, N. Schoutteten, X. Z. Liu, T. H. Li, Y. J. Yao, X. Y. Zhu, A. Q. Liu, G. J. Li, M. Z. Zhang, Z. L. Ling, B. Cao, V. Antonín, T. Boekhout, B. D. B. da Silva, E. De Crop, C. Decock, B. Dima, A. K. Dutta, J. W. Fell, J. Geml, M. Ghobad-Nejhad, A. J. Giachini, T. B. Gibertoni, S. P. Gorjón, D. Haelewaters, S. H. He, B. P. Hodkinson, E. Horak, T. Hoshino, A. Justo, Y. W. Lim, N. Menolli Jr., A. Mešić, J. Moncalvo, G. M. Mueller, L. G.
Nagy, R. H. Nilsson, M. Noordeloos, J. Nuytinck, T. Orihara, C. Ratchadawan, M. Rajchenberg, A. G. S. Silva-Filho, M. A. Sulzbacher, Z. Tkalčec, R. Valenzuela, A. Verbeken, A. Vizzini, F. Wartchow, T. Z. Wei, M. Weiß, C. L. Zhao y P. M. Kirk. 2019. Notes, outline and divergence times of Basidiomycota. Fungal Diversity 99: 105-367. DOI: https://doi.org/10.1007/ s13225-019-00435-4

Index Fungorum. 2021. Index Fungorum, base de datos. http:// www.indexfungorum.org/names/names.asp (consultado junio de 2021).

Keller, G. y J. F. Ammirati. 1983. Chemotaxonomic significance of anthraquinone derivatives in North American species of Dermocybe, section Sanguineae. Mycotaxon 18: 357-377.

Kong, A., A. Montoya, S. García-de Jesús, A. Ramírez-Terrazo, R. Andrade, F. Ruan-Soto, M. M. Rodríguez-Palma y A. Estrada-Torres. 2018. Hongos ectomicorrizógenos del Parque Nacional Lagunas de Montebello, Chiapas. Revista Mexicana de Biodiversidad 89(3): 741-756. DOI: http://doi. org/10.22201/ib.20078706e.2018.3.2527

Kornerup, A. y J. H. Wanscher. 1978. Methuen Handbook of Colour. 3rd. ed. Eyre Methuen. London, UK. 251 pp.

Largent, D. L., D. Johnson y R. Watling. 1977. How to identify mushrooms to genus III: microscopic features. 3rd ed. Eureka: Mad River Press Inc. Eureka, USA. 148 pp.

Lodge, D. J., J. F. Ammirati, T. O. Dell y G. M. Mueller. 2004. Terrestrial and lignicolous macrofungi: collecting and describing macrofungi. In: Mueller, G., G. F. Bills y M. S. Foster (eds.). Biodiversity of fungi: Inventory and monitoring methods. Elsevier Academic Press. New York, USA. Pp. 128158.

Padilla-Velarde, E. E., G. Zarco-Velaz, L. Guzmán-Dávalos y R. Cuevas-Guzmán. 2016. Primera contribución al conocimiento de macromicetes de la vertiente Norte del Cerro El Cípil, en la Costa Sur De Jalisco. Acta Botanica Mexicana 114: 137-167. DOI: https://doi.org/10.21829/ abm114.2016.1105

Pardave Díaz, L. M. 1996. Hongos venenosos del estado de Aguascalientes. Investigación y Ciencia (Universidad Autónoma de Aguascalientes) 18: 38-44.

Pérez-Silva, E., T. Herrera y A. Ocampo López. 2011. Nuevos registros de macromicetos para el municipio de Temascaltepec, Estado de México. Revista Mexicana de Micología 34: 23-30. 
Pérez-Silva, E., M. Esqueda y T. Herrera. 2008. Macromicetos tóxicos de Sonora, México. Revista Mexicana de Micología 28: 81-88.

Plants of the World. 2021. Plants of the World online. http:// www.plantsoftheworldonline.org/ (consultado junio de 2021).

QGIS Development Team. 2022. QGIS Geographic Information System v. 3.22.3. Open Source Geospatial Foundation. http://qgis.org (consultado enero de 2022).

Rinaldi, A. C., O. Comandini y T. W. Kuyper. 2008. Ectomycorrhizal Fungal diversity: separating the wheat from the chaff. Fungal Diversity 33: 1-45.

Rodríguez-Alcántar, O., D. Figueroa-García y M. J. HerreraFonseca. 2018. Catálogo de los hongos del Volcán de Tequila, municipio de Tequila, Jalisco, México. Polibotánica 45: 15-33. DOI: https://doi.org/10.18387/polibotanica.45.3

Smith, S. E. y D. J. Read. 2008. Mycorrhizal Symbiosis. 3rd ed. Academic. London, UK. 787 pp

Tedersoo, L., T. W. May y M. E. Smith. 2010. Ectomycorrhizal lifestyle in fungi: global diversity, distribution, and evolution of phylogenetic lineages. Mycorrhiza 20: 217-263. DOI: https://doi.org/10.1007/s00572-009-0274-x

Wijayawardene, N. N., K. D. Hyde, L. K. T. Al-Ani, L. Tedersoo, D. Haelewaters, K. C. Rajeshkumar, R. L. Zhao, A. Aptroot, D. V. Leontyev, R. K. Saxena, Y. S. Tokarev, D. Q. Dai, P. M. Letcher, S. L. Stephenson, D. Ertz, H. T. Lumbsch, M. Kukwa, I. V. Issi, H. Madrid, A. J. L. Phillips, L. Selbmann, W. P. Pfliegler, E. Horváth, K. Bensch, P. M. Kirk, K. Kolaříková, H. A. Raja, R. Radek, V. Papp, V. Dima, J. Ma, E. Malosso, S. Takamatsu, G. Rambold, P. B. Gannibal, D. Triebel, A. K. Gautam, S.
Avasthi, S. Suetrong, E. Timdal, S. C. Fryar, G. Delgado, M. Réblová, M. Doilom, S. Dolatabadi, J. Pawłowska, R. A. Humber, R. Kodsueb, I. Sánchez-Castro, B. T. Goto, D. K. A. Silva, F. A. de Souza, F. Oehl, G. A. da Silva, I. R. Silva, J. Błaszkowski, K. Jobim, L. C. Maia, F. R. Barbosa, P. O. Fiuza, P. K. Divakar, B. D. Shenoy, R. F. Castañeda-Ruiz, S. Somrithipol, A. A. Lateef, S. C. Karunarathna, S. Tibpromma, P. E. Mortimer, D. N. Wanasinghe, R. Phookamsak, J. Xu, Y. Wang, F. Tian, P. Alvarado, D. W. Li, I. Kušan, N. Matočec, S. S. N. Maharachchikumbura, M. Papizadeh, G. Heredia, F. Wartchow, M. Bakhshi, E. Boehm, N. Youssef, V. P. Hustad, J. D. Lawrey, A. L. C. M. A. Santiago, J. D. P. Bezerra, C. M. SouzaMotta, A. L. Firmino, Q. Tian, J. Houbraken, S. Hongsanan, K. Tanaka, A. J. Dissanayake, J. S. Monteiro, H. P. Grossart, A. Suija, G. Weerakoon, J. Etayo, A. Tsurykau, V. Vázquez, P. Mungai, U. Damm, Q. R. Li, H. Zhang, S. Boonmee, Y. Z. Lu, A. G. Becerra, B. Kendrick, F. Q. Brearley, J. Motiejūnaitè, B. Sharma, R. Khare, S. Gaikwad, D. S. A. Wijesundara, L. Z. Tang, M. Q. He, A. Flakus, P. Rodriguez-Flakus, M. P. Zhurbenko, E. H. C. McKenzie, M. Stadler, D. J. Bhat, J. K. Liu, M. Raza, R. Jeewon, E. S. Nassonova, M. Prieto, R. G. U. Jayalal, M. Erdoğdu, A. Yurkov, M. Schnittler, O. N. Shchepin, Y. K. Novozhilov, A. G. S. Silva-Filho, P. Liu, J. C. Cavender, Y. Kang, S. Mohammad, L. F. Zhang, R. F. Xu, Y. M. Li, M. C. Dayarathne, A. H. Ekanayaka, T. C. Wen, C. Y. Deng, O. L. Pereira, S. Navathe, D. L. Hawksworth, X. L. Fan, L. S. Dissanayake, E. Kuhnert, H. P. Grossart y M. Thines. 2020. Outline of Fungi and fungus-like taxa. Mycosphere 11(1): 1060-1456. DOI: https://doi.org/10.5943/mycosphere/11/1/8 